\title{
¿QUIÉNES SON TITULARES DE DERECHOS HUMANOS? LIBORIO HIERRO Y EL PREJUICIO ESPECIEÍSTA
}

\author{
Pablo de Lora \\ Universidad Autónoma de Madrid
}

\section{INTRODUCCIÓN}

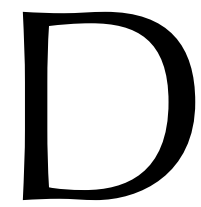

e la vasta producción intelectual de Liborio HIERRO destacan sin duda sus trabajos sobre derechos humanos, desde su artículo de 1982 «¿Derechos humanos o necesidades humanas? Problemas de un concepto» ${ }^{1}$ hasta su reciente libro ${ }^{2}$ en el que desgrana una concepción completa y sistemática de la justicia articulada en torno al ideal de los derechos humanos ${ }^{3}$.

Uno de los cimientos de ese edificio es el relativo a la titularidad de los derechos, y más específicamente la posible consideración de los niños como sujetos de derechos humanos, otra preocupación característica del quehacer filosófico-jurídico de Liborio HIERRO $^{4}$. HIERRO defiende, dicho de manera muy sumaria, que el respeto a los derechos humanos es una condición necesaria de la existencia de un orden político justo, pues de otro modo se niega la dignidad que tienen por igual todos los seres humanos en su condición de agentes morales 5 .

Una caracterización tal, sin embargo, suscita la pregunta de si la propiedad misma de la agencia moral — ser capaces de tener preferencias de segundo orden- ${ }^{6}$ es una condición igualmente necesaria para ser titular de tales derechos. Si lo es - como han sostenido tradicionalmente los partidarios de la llamada «teoría de la voluntad»

1 Publicado en la revista Sistema (núm. 46 de 1982). Merece también destacarse su compilación de textos junto con G. PeCES-BARBA, Textos básicos sobre derechos bumanos (Madrid, Universidad Complutense, 1973).

2 Los derechos humanos. Una concepción de la justicia, Marcial Pons, 2016.

3 Esta construcción ya se atisbaba en su contribución «El concepto de justicia y la teoría de los derechos», en J. L. Colomer y E. Díaz (dirs.), Estado, justicia, derechos (Madrid, Alianza, 2002).

4 «ंTienen los niños derechos? Comentario a la Convención sobre los Derechos del Niño», Revista de Educación, núm. 294, 221-233.

${ }^{5}$ Los derechos humanos son derechos morales subjetivos, esto es, posiciones normativas que permiten al individuo desplegar su autonomía, pero tienen carácter especial como derechos morales en tanto en cuanto requieren protección jurídica y sirven de criterio de legitimidad del orden jurídico-político.

${ }^{6}$ De acuerdo con la caracterización de BAYÓN que HIERRO asume (cit., 153). La propiedad de la agencia moral, como es bien sabido, se cifra en la idea de «personalidad» que HIERRO a su vez desagrega en tres atributos: autonomía, responsabilidad y autoconciencia; vid. Los derechos bumanos, op. cit., 139.

7 Ibid., 142-145. 
produce la, en términos de Carl WeLLMAN, «conclusión monstruosa» de que los niños no tendrían derechos humanos ${ }^{8}$. La evitación de ese indeseable corolario pasa, como se sabe, por suscribir la llamada «teoría del interés o del beneficiario». Pero entonces, advierte HIERRO, se genera un problema inverso de sobre-inclusión. En sus propias palabras: «Si nuestra teoría [del interés] permite atribuir derechos morales a individuos que carecen de agencia moral, porque se trata de garantizar la satisfacción de sus necesidades básicas para subsistir y realizarse [...] entonces ¿por qué no reconocer esos derechos en favor de entidades o individuos que pueden llegar a ser humanos (los fetos) o que han sido humanos (los muertos) o que se componen de seres humanos (los grupos) o que simplemente no son humanos (los animales, las plantas, la naturaleza)? Podríamos hacerlo en el ámbito de la moral, como lo hace el legislador en el ámbito del derecho. ¿Por qué damos tanta importancia moral a los derechos "humanos" y no a los derechos morales de cualquier cosa? Sin duda alguna en eso consiste el "prejuicio de especie" $\gg$.

En lo que sigue voy a discutir sucintamente el modo en el que Liborio HIERRO ha abordado ese prejuicio en su última obra para lo cual dividiré mi ensayo en tres apartados. En la sección 2 argüiré que el prejuicio de especie, tal y como es expuesto por HIERRO, no está bien caracterizado y que su propuesta puede ser mejor entendida a la luz del «personismo» defendido por Shelly KAGAN (sección 3). En la sección 4 analizaré su tesis de acuerdo con la cual la extensión del lenguaje de los derechos a los animales no humanos (en adelante $\mathrm{ANH}$ ) es contraproducente e inadecuada en tanto en cuanto no les debemos «justicia», y me preguntaré si no cabría concebir a algunas de las especies animales como miembros de nuestra comunidad política al igual que hacemos con los miembros «no paradigmáticos» de nuestra especie.

\section{ESPECIEÍSMO: EL PREJUICIO SOBRE EL «PREJUICIO DE ESPECIE»}

Hay un cierto prejuicio en el modo en el que Liborio HIERRO identifica el prejuicio de especie. No en la acepción atribuida por el diccionario de la RAE a la acción de prejuzgar, sino en el de no haber deslindado más cuidadosamente los distintos «sujetos problemáticos» de derechos humanos a la luz de la teoría del interés. El prejuicio de especie, dicho lisa y llanamente, se suscita en relación con todos aquellos entes de los que se pueda predicar que tienen intereses pero no pertenecen a la especie humana y solo por esta última razón son excluidos del círculo de sujetos de derechos humanos (cuando resulta que tener intereses es el factor moralmente relevante para la atribución de aquellos) ${ }^{10}$.

El resto de los candidatos a formar parte del universo de los titulares de derechos humanos que menciona HIERRO, y a los que comúnmente se niega tal condición, no sufren por la exclusión prejuicio de especie alguno. Es claro que ni los grupos (hu-

${ }^{8}$ Ibid., 149.

9 Ibid., 150.

10 Se incurre, entonces, en el vicio del «especieísmo». Para un tratamiento profundo e iluminador del mismo, vid. Ó. HORTA, «What is Speciesism?», Journal of Agricultural and Environmental Ethics, vol. 23, 2010, 243-266. 
manos), ni los muertos (humanos), ni los fetos (humanos) al menos en sus primeros estadios de desarrollo, tienen intereses ni necesidades y es por ello por lo que no son sujetos de derechos. ¿Qué nos permitiría afirmar tal cosa?

En este punto - la dilucidación de las nociones de interés y necesidad- Liborio HiERRO es ambiguo. En algún momento señala que de algunos primates superiores se puede discutir «[1] si cabe predicar [de ellos] las mismas características de agencia [que los seres humanos]» ${ }^{11}$; o [2] que algunos animales hayan llegado o puedan llegar a adquirir «el mínimo grado de autoconciencia necesario como para considerarlos agentes morales y reconocerles derechos morales fundamentales de idéntico peso a los derechos humanos» ${ }^{12}$. Sin embargo, en otro pasaje, criticando a Peter SINGER y Tom REGAN, sostiene que ambos habrían errado al «manejar una noción de interés» que no está ligada a la conciencia de ese interés. En esa carencia, sostiene HIERRO, los animales se parecen mucho a las plantas ${ }^{13}$.

La idea de «necesidad» remite tanto al medio causalmente eficaz para el logro de un fin, cuanto a la forma de satisfacer un interés o un deseo. Así, en el primer caso, podemos decir con sentido que «la planta necesita agua», y, en el segundo, que «necesito que me llames cuanto antes». En el primer supuesto no hay compromiso alguno con la existencia de estados mentales por parte del ente «necesitado», de la misma manera que afirmamos sensatamente la «necesidad de limpiar los filtros del aire acondicionado» (si queremos que funcione correctamente). La necesidad de recibir una llamada, sin embargo, sí entronca con la existencia de un deseo o interés que puede verse satisfecho o frustrado. Si este sentido de necesidad vinculado a la posesión de estados mentales es plausible, las plantas, como sostiene HIERRO, carecen de intereses porque, hasta donde sabemos, carecen de estados mentales ${ }^{14}$. De muchísimas especies animales, sin embargo, sí cabe predicar la existencia de esos estados ${ }^{15}$ y solo de muy pocos de ellos, por lo que sabemos, se podría decir, además, que tienen autoconciencia ${ }^{16}$. Bajo estos presupuestos, la teoría del interés bien podría caracterizar como titulares de de-

11 Los derechos bumanos, op. cit., 152.

12 Ibid., 155

13 Ibid., 153-154.

14 S. KagAN («What's Wrong With Speciesism?», Journal of Applied Philosophy, vol. 33, núm. 1, febrero de 2016, 1-21, 7) y M. KRAmER («Do Animals and Dead People Have Legal Rights?», Canadian Journal of Law and Jurisprudence, vol. 14, núm. 1, enero de 2001, 29-54, 33) entre otros, han defendido, por el contrario, que es plausible sostener que las plantas tienen intereses, y el segundo, incluso, que también tienen intereses los seres inanimados. Aunque sería interesante pormenorizar y discutir sus razones, excedería de los límites de este ensayo hacerlo. Es, por otro lado, innecesario dados los modestos objetivos que persigo.

15 El proceso cognitivo primario que, de acuerdo con los etólogos, caracteriza la sentencia implica «awareness» o «cognición consciente» de los estímulos internos y externos, es decir, la «capacidad de generar una escena mental en la que la información diversa se integra con el propósito de dirigir la conducta [...] la conciencia o sentiencia en los animales es un fenómeno que puede inferirse mediante el examen de las capacidades cognitivas y las características neuroanatómicas que un animal posee» (K. P. CHANDroO, I. J. H. DunCAN y R. D. MocCIA, «Can fish suffer?: perspectives on sentience, pain, fear and stress», Applied Animal Behaviour Science, vol. 86, 2004, 225-250, esp. 226-227).

${ }_{16} \mathrm{Y}$ de ninguno de ellos, ni siquiera de los grandes simios, que se acerquen al nivel de sofisticación cognitiva que atribuimos a quienes caracterizamos como agentes morales, esto es, aquellos que son capaces de tener preferencias sobre las propias preferencias. De la vasta literatura científica más reciente sobre toda esta materia vid. F. DE WAAL quien, en «The Tower of Morality», señala: «El deseo de un marco moral internamente consistente es singularmente humano. Somos los únicos a quienes nos preocupa por qué pensamos lo que pensamos [...]. Todo esto es mucho más abstracto que el concreto nivel conductual al que otros animales parecen operar» 
rechos a muchos $\mathrm{ANH}$, pero en ningún caso a las plantas, los artificios o la naturaleza en su conjunto.

\section{ANIMALES Y PERSONAS: CÓMO NO SER ESPECIEÍSTA}

Cuestión distinta es que esa forma de discriminación de los ANH —el especieísmo- equivalga a otras formas tradicionales y oprobiosas de discriminación, señaladamente al sexismo o al racismo. HIERRO lo niega puesto que al fijar como «presupuesto necesario para fundamentar los derechos humanos» la condición de ser agente moral no se está produciendo una desigualdad en función de un rasgo moralmente irrelevante (lo que hace, ahora sí, odioso al racismo o sexismo) ${ }^{17}$.

En una línea parecida se ha manifestado recientemente Shelly KAGAN: incluso aceptando la condición de pacientes morales (capaces de sentiencia) de los ANH, no es prejuiciosamente especieísta afirmar que el sufrimiento de los seres humanos «cuenta más» ${ }^{18}$.

Pero en realidad sí se produce tal discriminación, porque los intereses de los seres humanos, independientemente de su condición, cuentan siempre más que los intereses de los $A N H^{19}$. La discriminación se evita si la membrecía en la especie humana deja de considerarse relevante, manteniéndose, en cambio, la condición de ser persona (agente moral). De ese modo la posición no sería ya especieísta sino justificadamente «personista» como ha defendido KAGAN ${ }^{20}$. Pero, entonces, la «conclusión monstruosa» se nos cuela por la ventana: los bonobos y E. T. serían sujetos de derechos humanos, pero no los seres humanos con discapacidades cognitivas muy severas. La solución, para KAGAN, radica en adoptar una forma «modal» de personismo de acuerdo con la cual lo importante es la pertenencia a una especie en la que sus miembros son típicamente agentes morales: el individuo con una severa discapacidad cognitiva que le impide la agencia moral podría haber sido persona ${ }^{21}$. Tal personismo no incurriría en especieísmo porque atribuiría esa condición también a los que, no siendo seres humanos, pertenecen a una categoría en la que lo «normal» es ser persona. Supongamos, nos sugiere KAGAN, que los perros fueran originalmente de Marte y que allí se desarrollarán como personas pero no así en la Tierra porque, pongamos, la diferente fuerza de la gravedad

[en S. Macedo y J. Ober (eds.), Primates and Philosophers. How Morality Evolved, Princeton University Press, 2006, 161-181, 174].

17 HIERRO, Los derechos humanos, op. cit., 152.

18 Esa preferencia puede revelar además una muy «humana» (i. e. justificada) parcialidad hacia los miembros de nuestra propia especie, como sostuvo póstumamente B. Williams; vid. «The Human Prejudice», en A. W. Moore (ed.), Philosophy as a Humanistic Discipline, Princeton-Oxford: Princeton University Press, 2006, 149-152.

${ }_{19}$ El especieísmo también podría darse en el sentido inverso, esto es, la mera circunstancia de no ser miembros de nuestra especie habría de impedir ingresar en el club de los titulares de derechos humanos a entes con iguales o superiores capacidades cognitivas a nosotros.

${ }^{20}$ Cit., 9-11.

${ }^{21}$ Un argumento semejante puede ser rastreado, al menos, hasta un trabajo de C. COHEN, «The Case for the Use of Animals in Biomedical Research», en R. M. BAIRD y S. E. RosenBAUm (eds.), Animal Experimentation. The Moral Issues, Amherst, NY, Prometheus Books, 1991, 103-114, 106, y más recientemente M. S. LiAO, «The Basis of Human Moral Status», Journal of Moral Philosophy, vol. 7, núm. 2, 2010, 159-179. 
lo impide. Así y todo les deberíamos tratar como personas, de la misma manera que hacemos con los humanos con cognición severamente limitada ${ }^{22}$. No obstante, hay casos no-paradigmáticos de individuos que pertenecen a la especie humana que, no por accidente sino por «esencia», nunca hubieran podido llegar a ser persona, como ocurre típicamente con los anencefálicos, con lo que no les alcanzaría la protección del «personismo modal» defendido por KAGAN ${ }^{23}$.

\section{DERECHOS HUMANOS, ANIMALES NO HUMANOS Y COMUNIDAD}

La no pertenencia a la especie humana, por tanto, no parece una buena razón para negar la condición de sujeto de derechos básicos a los ANH. Sin embargo, uno podría preguntarse para qué tenemos que incluir a dichos individuos en el seno de la comunidad de los titulares de derechos básicos. La pregunta es relevante, sobre todo, porque muchos de los que sostienen la teoría de la voluntad, o la teoría del interés en su versión no inclusiva, pueden perfectamente acoger un buen número de restricciones a las acciones que implican maltrato a los que no gozan ni de agencia moral ni de la pertenencia a la especie humana. Sobre ese presupuesto se ha de leer a HIERRO cuando afirma que: «No es ni necesario ni conveniente extender la titularidad de derechos morales de este tipo, fundamento de un orden justo, a ningún tipo de entidad individual o colectiva que no sea un agente moral o pertenezca a la misma especie que los agentes morales. La razón de esta conclusión se apoya simplemente en la función que el invento de los derechos humanos trataba, y sigue tratando, de cumplir» ${ }^{24}$. Y esa función es, como se apuntaba antes, proteger un ámbito de soberanía individual frente al poder físico o normativo de otros, y, paradigmáticamente, frente al orden político: los derechos son trumps (DwORKIN) o moral side-constraints (NOZICK). Por ello, prosigue HIERRO, los derechos humanos no constituyen ni tienen porque constituir una teoría moral completa. Los animales, el planeta, el patrimonio histórico-artístico deben ser protegidos y cuidados pero para ello: «No necesitamos devaluar el lenguaje de los derechos hasta hacerle perder su sentido y, lo que sería peor, su función en el diseño de un orden político justo» ${ }^{25}$.

Los derechos humanos, en la concepción de HIERRO, son la mejor forma de «dar a cada uno lo suyo», un desiderátum de la justicia y están vinculados a la conformación de un «orden» que permita la interacción mutuamente provechosa entre los individuos. Nuestros tratos con los ANH pertenecerían, en cambio, a una sucursal moral distinta: la del respeto, la compasión o la «reverencia», si acaso. Y ello no meramente, o no fundamentalmente, porque los ANH no sean «uno de los nuestros», sino porque con ellos no conformamos una, parafraseando a John RAWLS, «sociedad bien ordenada».

22 KAGAN, cit., 13-14.

23 J. McMahan, «On Modal Personism», The Journal of Applied Philosophy, vol. 33, núm. 1, febrero de 2016, 26-30, esp. 27-28. Por supuesto hay quienes, como David DEGRAZIA rechazan como criterio moralmente relevante el haber podido ser persona si resulta que sus capacidades son finalmente - ya sea por esencia o accidente- las mismas; vid. «Modal Personhood and Moral Status: A Reply to Kagan's Proposal», The Journal of Applied Philosophy, vol. 33, núm. 1, febrero de 2016, 22-25, esp. 24.

24 HiERro, Los derechos bumanos, op. cit., 155.

25 Ibid., 156. 
¿Es eso ciertamente así? De nuevo aquí podríamos hacer preguntas semejantes a las de los anteriores apartados: de las comunidades humanas no solo forman parte sujetos racionales y razonables — por seguir con el imaginario rawlsiano- sino también humanos no paradigmáticos. De los niños se podría decir que su inclusión deriva del hecho de que serán miembros plenamente cooperantes de la sociedad ${ }^{26}$. ¿Pero y los que en ningún caso llegarán a serlo por su condición de extrema discapacidad cognitiva? Esa circunstancia no les impide «estar entre nosotros» - solo entre nosotros pueden encontrar un mínimo florecimiento «humano»— aunque no sea en la condición de ciudadanía plena. Del mismo modo - y con esto no estoy estableciendo ninguna relación de semejanza sino una semejanza de relaciones- se encuentran los animales de compañía, los amansados, o los que, de maneras muy variadas —desde los perrosguía de los ciegos hasta los llamados «animales terapéuticos»- nos permiten vivir una vida mejor como seres humanos. ¿Es una excentricidad pensarles como miembros de nuestras sociedades políticas? ${ }^{27}$. Estoy inclinado a pensar que no, aunque nadie como Liborio HiERRO para sacarme de ese, y algún que otro, tropiezo filosófico-jurídico que haya podido cometer en este comentario.

${ }^{26}$ Los derechos, como expresión de la justicia, no actúan bajo el presupuesto de una comunidad estática, sino que la sociedad política se ordena con la vocación de perdurar en el tiempo.

27 Esta es la tesis desarrollada por W. Kymlicka y S. Donaldson en Zoopolis. A Political Theory of Animal Rights, Oxford, Oxford University Press, 2011. De la «ciudadanía animal» quedan excluidos, eso sí, los animales salvajes. 